\title{
Estrategias Metodológicas de la Educación Física para la Inclusión de Escolares con Mono-Para y Tetraplejia
}

\section{Methodological Strategies of Physical Education for the Inclusion of Schoolchildren with Mono-Para and Tetraplegia}

\author{
Edwin Geovanny Naranjo-Naranjo \\ edwin.naranjo.29@est.ucacue.edu.ec \\ Universidad Católica de Cuenca, Azogues \\ Ecuador \\ https://orcid.org/0000-0002-8391-6828 \\ Zoila Guillermina Torres-Palchisaca \\ ztorresp@ucacue.edu.ec \\ Universidad Católica de Cuenca, Azogues \\ Ecuador \\ https://orcid.org/0000-0003-3078-6465 \\ Gema Barrachina-Fernández \\ gema.barrachina@ucacue.edu.ec \\ Universidad Católica de Cuenca, Azogues \\ Ecuador \\ https://orcid.org/0000-0002-4341-3775
}

Recepción: 30 de agosto 2021

Revisado: 20 de septiembre 2021

Aprobación: 15 de noviembre 2021

Publicación: 01 de diciembre 2021 


\title{
RESUMEN
}

La investigación tiene como objetivo implementar estrategias metodológicas para la inclusión de estudiantes con discapacidad motriz en la Educación Física, para lo cual se abordó desde una investigación descriptiva Entre los datos más relevantes en la encuesta aplicada, se puede citar que; las actividades con mayor dificultad al momento de impartir sus clases de Educación Física dirigidos a estudiantes con discapacidad motriz son: la planificación y aplicación de estrategias de aprendizaje; de igual forma se evidencia que no cuentan con estrategias metodológicas aplicadas a la Educación Física para trabajar idóneamente en estudiantes con discapacidad motriz. Durante la investigación se han cumplido los objetivos propuestos y además, se ha podido conocer la realidad educativa en estudiantes con NEE, implementando de esta forma algunas estrategias metodológicas que podrán los docentes aplicar a estudiantes con discapacidad motriz.

Descriptores: Aprendizaje activo; método de aprendizaje; educación física. (Palabras tomadas del Tesauro UNESCO).

\begin{abstract}
The objective of the research is to implement methodological strategies for the inclusion of students with motor disabilities in Physical Education, for which it was approached from a descriptive investigation Among the most relevant data in the applied survey, it can be mentioned that; The activities with the greatest difficulty at the time of teaching their Physical Education classes aimed at students with motor disabilities are: the planning and application of learning strategies; Likewise, it is evident that they do not have methodological strategies applied to Physical Education to work ideally with students with motor disabilities. During the investigation, the proposed objectives have been fulfilled and, in addition, it has been possible to know the educational reality in students with SEN, thus implementing some methodological strategies that teachers can apply to students with motor disabilities.
\end{abstract}

Descriptors: Activity learning; learning methods; physical education. (Words taken from the UNESCO Thesaurus). 


\section{INTRODUCCIÓN:}

La inclusión educativa hoy en día debe ser un cimiento sólido para construir una enseñanza y aprendizaje significativo en la cual los estudiantes promuevan la igualdad y equidad entre ellos, respetando sus cualidades y características personales; de tal forma es importante establecer mecanismos que ayuden a conocer e identificar las estrategias metodológicas de la Educación Física para la inclusión de escolares con discapacidad motriz. Para esto es necesario entender a la inclusión desde una perspectiva totalmente amplia de aceptación a la diversidad.

"En la mayoría de los países, reconocen a la educación inclusiva como un motor fundamental para el desarrollo humano y cambio social", como lo afirma la (UNESCO, 1994) en la cita de (Pappous, 2015, p.108). En síntesis, para la UNESCO la educación inclusiva tiene como finalidad dar la oportunidad a toda la humanidad de ser parte del sistema escolar, acogiéndose al derecho humano básico de la educación, generando las bases necesarias para conseguir una sociedad más justa y solidaria, independientemente de sus características o dificultades personales y particulares.

En la sociedad actual; los estudiantes que adolecen algún tipo de discapacidad no son integrados de manera armónica dentro del sistema educativo, en donde las oportunidades no son igual a un estudiante común, de tal forma es obligación del estado ecuatoriano impulsar la transformación de la enseñanza con la aspiración de consolidar la formación especial en el marco de la normativa legal vigente, en este sentido, la educación en el Ecuador cuenta con un amplio marco normativo sólido que impulsa la implementación de políticas públicas, alineadas con los acuerdos y tratados internacionales, como lo expresan (Tomala \& Valverde 2001).

En base a lo anterior, la vicepresidencia en su Módulo 1 Educación inclusiva y especial (2011) establece que, la educación inclusiva va dirigido de manera implícita al derecho de recibir una educación con eficiencia y de calidez, por lo cual el objetivo principal es de incluir a toda la población de estudiantes a las escuelas regulares, consolidando el 
proceso de participación y aprendizaje, con miras a que el Ecuador cumpla con el proceso de transformación hacia una educación equitativa e inclusiva que ayude, fortalezca y de solución a los problemas actuales que sufre la educación incluyente.

Bajo estos parámetros es necesario manifestar que; para que exista un cumplimiento total al proceso de transformación educativa a la inclusión, es preciso contar con estrategias metodológicas aplicadas a estudiantes con discapacidad motriz, con el propósito de mejorar la enseñanza aprendizaje de la Educación Física, de esta forma se pretende que el discente pueda aproximarse a los contenidos curriculares de la asignatura.

En síntesis; es de vital importancia monitorear y contribuir al cumplimiento de la normativa legal; en la cual los docentes dentro del proceso inclusivo cumplen con un rol fundamental, de modo que es obligación recibir y atender a estudiantes con necesidades educativas especiales (NEE) dentro de sus clases regulares y por supuesto conocer las estrategias metodológicas de cómo aplicar las diferentes adaptaciones curriculares; con el fin de que los estudiantes se encuentren en posiciones de igualdad dentro del proceso educativo, de tal forma que el docente se apodere de la formación -actualización desde y para la inclusión educativa.

Ante lo expuesto se plantea el objetivo de investigación; que tiene como propósito facilitar una guía de estrategias metodológicas con actividades que puedan aplicar los docenes de Educación Física, a estudiantes con discapacidad motriz, específicamente aquellos que padecen de: monoplejia, paraplejia, tetraplejia; aplicable al sistema escolar ecuatoriano.

\section{Referencial Teórico}

La presente investigación se fundamenta en establecer los principales referentes teóricos y metodológicos de la Educación Física para la inclusión de escolares con monoplejia, paraplejia y tetraplejia, afecciones que se encuentran dentro de la discapacidad motriz. 
De ahí surge la necesidad de contar con un bagaje de acciones dirigidas a motivar, mantener e incluir la participación en los estudiantes con discapacidades motrices, en diversas actividades de la Educación Física. De tal forma; a continuación, se generan los principales elementos teóricos que sustentan las estrategias metodológicas para incluir a estudiantes con discapacidad motriz en el ámbito escolar.

\section{Tipos de discapacidad}

De acuerdo a Taboada (2012) la discapacidad incluye la "físico-motora, sensorial, visceral, cognitiva o intelectual, y discapacidad psíquica o mental. Desde el punto de vista causal, se clasifican según el momento de la vida en que se originó el defecto: prenatal, perinatal o postnatal". (p. 1)

Las discapacidades físicas se clasifican de acuerdo a varios criterios según autores, pero para efectos de la presente investigación se realiza la siguiente clasificación:

Discapacidad físico-motora: se refiere a "personas con parálisis de una extremidad superior o inferior, hemiplejia, paraplejia o tetraplejia, amputación de miembros superiores o inferiores, trastornos en la coordinación y en el tono muscular además trastornos graves del sistema nervioso" como lo afirma (Taboada \& Minaya,2012, p,01).

Discapacidad Intelectual: "personas con un funcionamiento intelectual significativamente inferior a la media, déficit en la conducta adaptativa, y comienzo en la infancia, o antes de los 18 años de edad" (Taboada \& Minaya, 2012, p.2).

Discapacidades físicas mixtas: este tipo de discapacidad es el más extenso, ya que aquí existe una diversidad de trastornos funcionales y que en muchos de los casos requieren de hospitalización e inmovilización, como lo manifiesta (Álava et al., 2019). La investigación realizada se fundamenta en la discapacidad física motriz según el número de extremidades y partes del cuerpo que la afectan: mono, para y tetraplejia.

Monoplejia: es un trastorno motor que afecta a un solo miembro y se divide en: monoplejia sin atrofia muscular que suele deberse a lesión de la primera neurona de la 
vía motora y la monoplejia con atrofia muscular puede deberse a desuso de la extremidad por una lesión central, como señala, El portal de contenidos en neurología, (2021, p.3). Paraplejia: "la paraplejia es la parálisis de los miembros inferiores cuando la lesión es completa, se afectan las vías motoras, sensitivas y autónomas, con pérdida de la sensibilidad somática y de la movilidad voluntaria", como lo expresa (Moreno \& Fergusson, 2009, p.2).

Tetraplejia: para Pascual, "la tetraplejia es una enfermedad permanente y no progresiva que afecta a la sensibilidad y movilidad de las cuatro extremidades y tronco, suele ir acompañada de otros trastornos o alteraciones en el sistema vegetativo y en las funciones" (2011, p.2).

Los estudiantes que adolecen algún tipo de discapacidad motriz como lo indica la clasificación presentada; tendrán derecho a recibir adaptaciones curriculares adaptadas de acuerdo a su necesidad, de igual forma el centro educativo que los acoja deberá cumplir con las normas básicas de adecuaciones y accesibilidad, de tal forma que los espacios deben ser abiertos y fáciles de transitar. De igual forma las rampas son indispensables para estudiantes con silla de ruedas o discapacidad motriz, sumándose a esto los inodoros que deben estar adaptados para personas con necesidades educativas especiales (NEE), como lo afirma Grzona (2014) "consideramos a la accesibilidad educativa como el despliegue de las acciones institucionales que eliminan los obstáculos y las barreras para posibilitar condiciones que reconocen el derecho a la individualidad de cada alumno". (p.140)

\section{Adaptaciones curriculares}

Para atender las diferentes discapacidades dentro del campo educativo, surge la necesidad de conocer e identificar que las adaptaciones curriculares son el instrumento fundamental para conseguir la individualización de la enseñanza, por cuanto son modificaciones que se realizan en la programación curricular común para entender las 
diferencias individuales del alumnado, convirtiéndose en medidas de flexibilización del currículo escolar, orientadas a posibilitar a estudiantes que están por debajo o por encima del promedio puedan participar y beneficiarse de la enseñanza, como lo afirman (Navarro et al., 2016).

Ante lo enunciado en la Guía de Trabajo Adaptaciones Curriculares para la educación especial e inclusiva (2013), los docentes son los protagonistas principales al momento de realizar las adaptaciones curriculares, los mismos que trabajan en forma articulada con el Departamento de Consejería Estudiantil u organismos que dentro de cada Institución educativa realizan estas coordinaciones, ante lo cual es necesario tener un diagnóstico médico actualizado del estudiante, pudiendo ser este: físico, médico, intelectual y terapéutico. La única persona encargada de validar estos documentos es la autoridad competente, en tanto que los padres de familia y/o representantes legales cooperarán con la información de datos y deberán realizar un oficio de aceptación por escrito, en la cual indique su aprobación con la adaptación curricular implementada para su representado.

\section{Estrategias metodológicas}

Para Díaz, V. (2021) en la cita (Petrou \& Henríquez, 2006, p.145), "la estrategia metodológica es un elemento fundamental del proceso de enseñanza- aprendizaje, puesto que de ella depende la orientación y operatividad del proceso e involucra una interrelación constante con los contenidos, objetivos, destrezas con criterio de desempeño". En consecuencia, y de acuerdo a Gutiérrez et al., "las estrategias metodológicas determinan la forma de llevar a cabo un proceso didáctico, brindan claridad sobre cómo se guía el desarrollo de las acciones para lograr los objetivos, en el ámbito educativo" (2018, p.38). 


\section{Estrategias activas aplicadas a estudiantes con NEE, en la Educación Física.}

En la actualidad la Educación Física, ha generado varios cambios, desde la concepción y enfoque de la signatura por parte de los estudiantes, lo que lleva a pensar en nuevas formas de abordar los procesos de enseñanza; de tal forma es necesario indicar que las estrategias tradicionales no permiten dar respuesta a las necesidades formativas de las nuevas generaciones, dada la diversidad y requerimientos de los estudiantes actuales. Por lo tanto, es pertinente identificar y describir estrategias activas eficaces que promueven el logro social y académico en diferentes niveles y contextos de aprendizaje.

Aprendizaje Cooperativo: para Velázquez \& López (2014) en el aprendizaje cooperativo es importante rescatar que el estudiante es responsable de su propio aprendizaje, pero también y muy fundamentalmente, por el de todos y cada de uno de sus compañeros de clase. De igual forma a juicio de Barros (2021) el aprendizaje cooperativo se convierte en una estrategia de enseñanza - aprendizaje donde la idea principal es que los estudiantes aprenden unos de otros, generando aspectos innovadores de aprendizaje social y académico, siendo una nueva forma de enseñar y promover los aprendizajes interpersonales.

Aprendizaje Basado en Problemas : el ABP se realiza a través del planteamiento de un problema por parte de los docentes, en el que la finalidad no es resolver el problema; al contrario este es utilizado como una guia para identificar los temas de aprendizaje, los mismos que serviran para el estudio del tema de manera independiente o grupal. La parte esencial del ABP implica tres lineamientos fundamentales: confrontar el problema, realizar estudio independiente y regresar al problema, como lo argumenta (Mendez et al.,2018).

Flipped Classroom o aula invertida: para Escobar (2020) esta nueva y valiosa técnica se presenta como la respuesta a grandes desafíos al cual estamos viviendo hoy en día como es la tecnología, con el propósito de aprender haciendo y no memorizando, donde 
los estudiantes son protagonistas de su propio aprendizaje dentro y fuera de las aulas, además este nuevo reto cuenta con plataformas digitales en las cuales los dicentes podrán interactuar con sus compañeros a través de videos, poscart, foros, entre otros.

Gamificacion: en el apogeo de las Tecnologías de la Información y la Comunicación surge una nueva didáctica basada en los videos juegos implementados a la educación, con esta nueva técnica se pretende mejorar el interés y el apremio de los estudiantes por las asignaturas, la motivación por aprender jugando y los diferentes retos que implica estos videos juegos. (Lobo Canella, 2018).

\section{MÉTODO}

La investigación se realizó en primera instancia a través de una revisión bibliográfica de artículos científicos en revistas indexadas de diferentes cuartiles con la ayuda del buscador y Google Scholar, Scopus, Web of Science, Scielo, Redalyc, y Dialnet, que proporcionaron el conocimiento sobre investigaciones ya existentes sobre el tema de Estrategias metodológicas de la Educación Física para la inclusión de escolares con mono-para y tetraplejia (Erazo, 2021).

El diseño del presente estudio se enfoca a una investigación cuantitativa de tipo descriptiva con corte transversal y enfoque mixto (Erazo y Narváez, 2020), se aplicó un muestreo no probabilístico voluntario, la misma que fue dirigida a docentes del área de Educación Física de la Ciudad de Cuenca, en la que manifestaron su voluntad de responder al cuestionario estando de acuerdo con las condiciones del estudio, esta se realizó de forma no aleatoria por conveniencia y la estimación mínima de participaciones para una muestra representativa fue de 201 docentes del área.

Como medio de recolección se utilizó la encuesta con 13 Ítems, utilizando la escala Likert, el proceso de revisión y validación de cada una de las preguntas del cuestionario y su correspondencia con los objetivos lo realizaron especialistas en el área de Educación Física con más de 10 años de experiencia, maestría, elaboración y dirección de artículos. 
La encuesta fue realizada vía on line (google forms) y el proceso de la información recabada se efectuó a través de una revisión sistemática de resultados, utilizando el paquete estadístico SPSS versión 19 IBM, aplicando un análisis descriptivo de frecuencias y porcentajes para las variables cualitativas, quedando excluidos los sujetos que no contestaron algunos ítems del test o lo hicieron de forma incorrecta. La aplicación del Alfa de Cronbach permitió medir la fiabilidad y consistencia interna de los ítems que componen el instrumento, el valor obtenido fue de 0,867 lo cual demuestra una consistencia alta.

Los métodos teóricos utilizados fueron; el análisis documental para obtener información de diferentes bases de datos académicos relacionado al tema de investigación; el método analítico- sintético permitió descomponer en su totalidad los elementos que se vinculan con las estrategias metodológicas para estudiantes con discapacidad motriz y comprender sistemáticamente sus efecto y relaciones. De igual manera, se empleó el inductivo- deductivo a través de cual se analizaron las partes y relaciones de la temática desde un enfoque particular a lo general hasta llegar a conclusiones específicas.

\section{RESULTADOS}

\section{Tabla 1.}

Resultados de la encuesta realizada a docentes de Educación Física sobre "Estrategias metodológicas de la Educación Física para la inclusión de escolares con mono-para y tetraplejia".

\begin{tabular}{llll}
\hline 1. Genero & & Frecuencia & Porcentaje \\
\hline & Mujer & 86 & 42,8 \\
& Hombre & 115 & 57,2 \\
& Total & 201 & 100,0 \\
\hline 2.Edad & & \\
\hline & $20-25$ años & 13 & 6,5 \\
& $26-30$ años & 39 & 19,4 \\
& $31-35$ años & 40 & 19,9
\end{tabular}


Revista Arbitrada Interdisciplinaria KOINONIA

Año VI. Vol VI. N4. Edición Especial: Educación III. 2021

Hecho el depósito de Ley: FA2016000010 ISSN: 2542-3088

FUNDACIÓN KOINONIA (F.K). Santa Ana de Coro. Venezuela.

Edwin Geovanny Naranjo-Naranjo; Zoila Guillermina Torres-Palchisaca; Gema Barrachina-Fernández
36 - 40 años
48
23,9
Más de 40 años
61
30,3
Total
201
100,0

3. ¿Cuál es su grado de preparación académica actual?

$\begin{array}{lcc}\text { Licenciado en Educación Física o } & 173 & 86,1 \\ \begin{array}{l}\text { afines } \\ \text { Maestría en Educación Fisca o afines }\end{array} & 28 & 13,9 \\ \text { Total } & 201 & 100,0\end{array}$

\section{4. ¿Cuántos años labora como docente en el área de Educación Física?}

$\begin{array}{lll}1 \text { a } 5 \text { años } & 16 & 8,0 \\ 6 \text { a } 10 \text { años } & 45 & 22,4 \\ 11 \text { a } 15 \text { años } & 55 & 27,4 \\ 16 \text { a } 20 \text { años } & 64 & 31,8 \\ 21 \text { a mas } & 21 & 10,4 \\ \text { Total } & 201 & 100,0\end{array}$

5. ¿De acuerdo a su experiencia elija tres actividades que realiza Usted cuando tiene un estudiante con Necesidades Educativas Especiales asociadas a la discapacidad motriz en sus clases de Educación Física?

\begin{tabular}{|c|c|c|}
\hline Autocapacitación & 64 & 25 \\
\hline $\begin{array}{l}\text { Me asesoro con un compañero } \\
\text { docente de mayor experiencia. }\end{array}$ & 31 & 17 \\
\hline Solicito apoyo en el DECE & 20 & 13 \\
\hline $\begin{array}{l}\text { Me apoyo en los padres del } \\
\text { estudiante con NEE. }\end{array}$ & 6 & 5 \\
\hline $\begin{array}{l}\text { Busco el expediente del estudiante } \\
\text { con NEE para conocer sus } \\
\text { potencialidades y diagnostico medico }\end{array}$ & 10 & 8 \\
\hline $\begin{array}{l}\text { Elaboro pruebas de diagnóstico } \\
\text { individualizadas }\end{array}$ & 15 & 12 \\
\hline
\end{tabular}


los estudiantes con NEE

Total

100,0

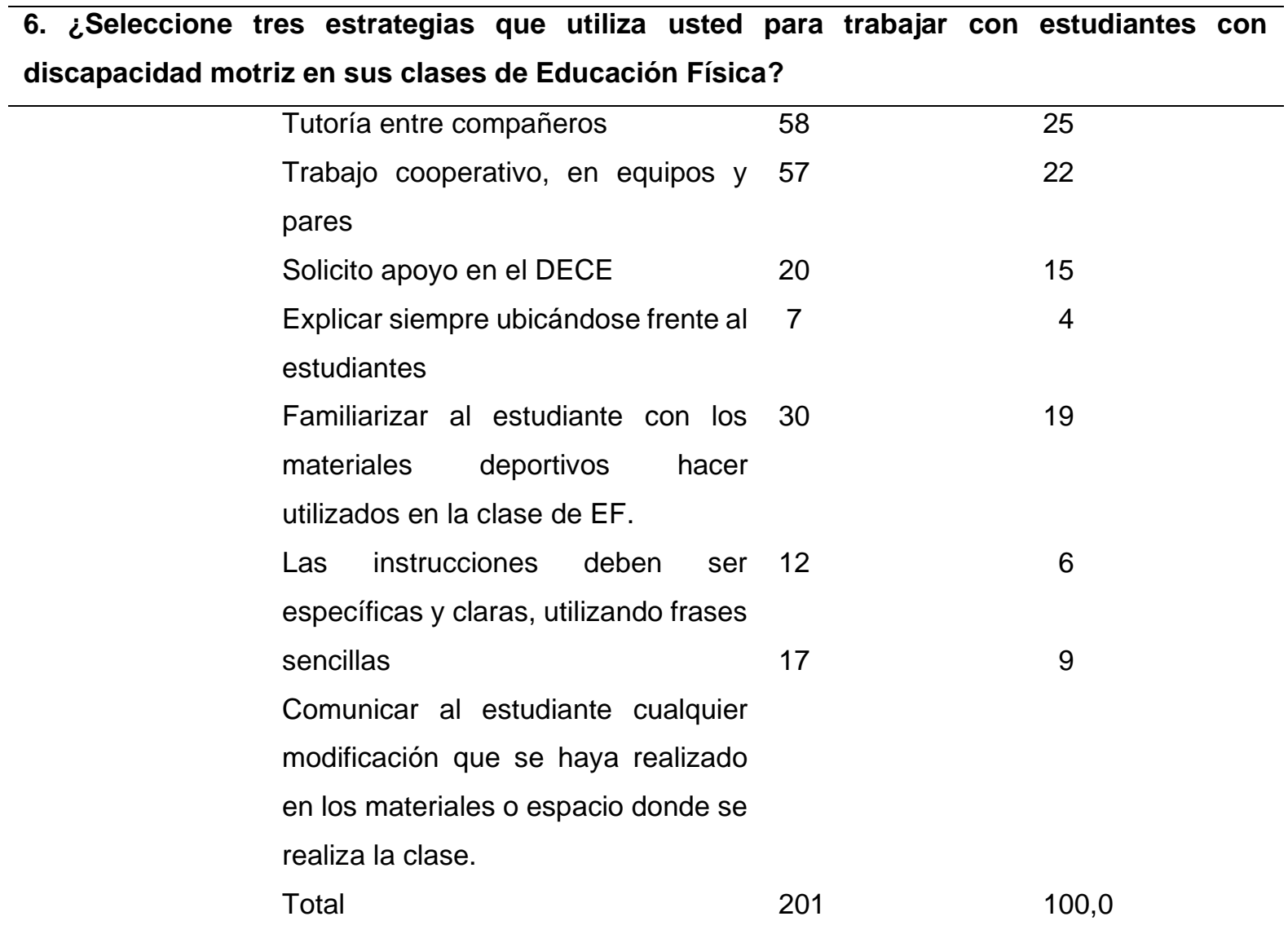

7. ¿Seleccione dos actividades, que más le dificulta a Usted al momento de impartir sus clases de Educación Física dirigidos a estudiantes con discapacidad motriz?

$\begin{array}{lll}\text { Planificación } & 60 & 29 \\ \text { Aplicar estrategias de aprendizaje } & 81 & 38 \\ \text { Insumos e infraestructura } & 38 & 22 \\ \text { Fomentar la equidad } & \text { y } 22 & 11 \\ \text { compañerismos } & & \\ \text { Total } & 201 & 100,0\end{array}$


8. ¿En su trayectoria como docente, con qué frecuencia usted trabaja con estudiantes que presentan Necesidades Educativas Especiales asociadas a la discapacidad motriz?

\begin{tabular}{lll}
\hline Nunca & 2 & 1,0 \\
Raramente & 60 & 29,9 \\
Ocasionalmente & 97 & 48,3 \\
Frecuentemente & 40 & 19,9 \\
Muy frecuentemente & 2 & 1,0 \\
Total & 201 & 100,0
\end{tabular}

9. ¿Cuenta Usted con estrategias metodológicas aplicadas a la Educación Física para trabajar idóneamente en estudiantes con discapacidad motriz?

$\begin{array}{lrl}\text { Nunca } & 99 & 49,3 \\ \text { Raramente } & 92 & 45,8 \\ \text { Ocasionalmente } & 7 & 3,5 \\ \text { Frecuentemente } & 3 & 1,5 \\ \text { Total } & 201 & 100,0\end{array}$

10. ¿El currículo de Educación Física 2016 incluye fundamentos, metodologías y estrategias específicas que se puedan aplicar en estudiantes asociados a la discapacidad motriz?

$\begin{array}{lcl}\text { Totalmente en desacuerdo } & 107 & 53,2 \\ \text { En desacuerdo } & 89 & 44,3 \\ \text { Ni de acuerdo ni en desacuerdo } & 2 & 1,0 \\ \text { De acuerdo } & 2 & 1,0 \\ \text { Totalmente de acuerdo } & 1 & , 5 \\ \text { Total } & 201 & 100,0\end{array}$

11. ¿En su institución educativa cumplen con las normas básicas y cuentan con los implementos necesarios para el desarrollo de las clases de Educación Física, para propiciar la participación armónica de estudiantes con necesidades educativas especiales asociadas a la discapacidad motriz?

$\begin{array}{lll}\text { Nunca } & 85 & 42,3 \\ \text { Casi Nunca } & 92 & 45,8 \\ \text { A veces } & 21 & 10,4\end{array}$




\begin{tabular}{|c|c|c|}
\hline Casi Siempre & 2 & 1,0 \\
\hline Siempre & 1 &, 5 \\
\hline Total & 201 & 100,0 \\
\hline \multicolumn{3}{|c|}{$\begin{array}{l}\text { 12. ¿Está de acuerdo que, para atender correctamente a un estudiante con discapacidad } \\
\text { motriz en una clase de Educación Física, sea necesario partir de un diagnóstico: médico, } \\
\text { físico e intelectual? }\end{array}$} \\
\hline Totalmente en desacuerdo & 3 & 1,5 \\
\hline En desacuerdo & 2 & 1,0 \\
\hline $\mathrm{Ni}$ de acuerdo ni en desacuerdo & 2 & 1,0 \\
\hline De acuerdo & 101 & 50,2 \\
\hline Totalmente de acuerdo & 93 & 46,3 \\
\hline Total & 201 & 100,0 \\
\hline \multicolumn{3}{|c|}{$\begin{array}{l}\text { 13. ¿Considera usted necesario que en su institución educativa cuente con una guía de } \\
\text { estrategias metodológicas en el área de Educación Física para aplicar a estudiantes con } \\
\text { discapacidad motriz? }\end{array}$} \\
\hline Poco necesario & 1 & ,5 \\
\hline Necesario & 86 & 42,8 \\
\hline Muy necesario & 114 & 56,7 \\
\hline Total & 201 & 100,0 \\
\hline
\end{tabular}

Fuente: Los autores.

\section{DISCUSIÓN}

El tamaño de la muestra de estudio fue de 201 docentes, en la cual, el 57,2\% corresponde al género masculino y el $42,8 \%$ al género femenino; la edad predominante fue de más de 40 años, con un grado académico de Licenciado en Educación Física y a fines; en cuanto a la experiencia profesional se registra entre 16 a 20 años $(31,8 \%)$ en la labor docente. Con respecto a la frecuencia de trabajo de los docentes con estudiantes que presentan Necesidades Educativas Especiales asociadas a la discapacidad motriz, se obtuvo que ocasionalmente $(48,3 \%)$ laboran con estudiantes con NEE en la educación regular, sin embargo, a pesar de ser un grupo minoritario de estudiantes, 
necesitan ser considerados dentro del proceso educativo de manera armónica, participativa, equitativa y constructiva, partiendo siempre de un diagnóstico médico, físico e intelectual, situación con la que concuerdan los docentes y que se refleja en el resultado de la pregunta 12 , es decir un 46,3\%.

Por otro lado, al consultar a los docentes que actividades realizan cuando tiene un estudiante con NEE asociados a la discapacidad motriz, un $25 \%$ de los encuestados indica que se auto capacita, aplica estrategias para mejorar el ambiente de la clase (20\%) y además se asesora con un compañero docente de mayor experiencia (17\%), en relación a los datos obtenidos Soto Calderón (2003) expresa que es necesario brindar una formación de calidad a los estudiantes que adolecen algún tipo de discapacidad, para lo cual el pedagogo deberá capacitarse constantemente, cumpliendo de esta manera el rol protagónico de la labor docente en el proceso educativo.

En el mismo sentido, se puede observar que la estrategia más utilizada por los docentes dentro de su clase es la tutoría entre compañeros (25\%), trabajo cooperativo en equipos y pares (22\%), con respectos a estos datos (Franco \& Ayala) en la cita de Antonio Campo Peña et al. (2020) manifiestan que, las estrategias correctamente aplicadas ayudan a mejorar de manera óptima las actividades de formación pedagógica de los estudiantes, de tal forma que facilitan a los docentes la enseñanza-aprendizaje de forma comprensiva y eficaz, para mejorar destrezas, habilidades y el desarrollo integral.

Un importante resultado arroja la pregunta número siete, en la cual los docente expresan que las actividades que más se les dificulta cuando imparte sus clases de Educación Física dirigidos a estudiantes con discapacidad motriz son: aplicar estrategias de aprendizaje (38\%) y la planificación con el 29\%, esto lo confirma los resultados obtenidos en la pregunta número nueve en la cual se observa que el 49,3\% no cuentan con estrategias metodológicas aplicadas a la Educación Física para trabajar idóneamente con estudiantes con discapacidad motriz, en tanto que apenas el 1,5\% de la población encuestada responde tener frecuentemente estrategias, al respecto, Dabdub \& Pineda 
(2015) consideran que, cuando el docente tenga en su clase estudiantes con necesidades educativas especiales asociadas o no a la discapacidad, se hallan frente al deber profesional y moral de: buscar, planificar y aplicar estrategias metodológicas que le permitan desarrollar un adecuado proceso de enseñanza y que propicien en cada uno de los estudiantes un aprendizaje significativo.

En lo que respecta a la pregunta diez el $53,2 \%$ de los encuestados precisan que están totalmente en desacuerdo, que en el currículo de Educación Física 2016 incluye fundamentos, metodologías y estrategias específicas que se puedan aplicar en estudiantes asociados a la discapacidad motriz y apenas un $0,5 \%$ responde estar totalmente de acuerdo, en virtud de los datos revelados, el Curriculo 2016 en su apartado referentes del ajuste curricular expresa que todos los docentes de educación general básica, incluidos docentes universitarios y asesoría nacional e internacional, desarrollaron una revisión exhaustiva del currículo en todos los niveles de educación obligatoria y analizando los aspectos cognitivos de los documentos curriculares, de cierta forma el currículo actual plantea una educación más amplia y flexible, con el firme propósito de brindar mejores opciones para la atención idónea a todos los estudiantes en todos los niveles del país, atendiendo a la diversidad entre ellos; sin embargo se evidencia que dentro del ajuste curricular no existen estrategias y metodologías específicas para aplicar en estudiantes con NEE y mucho menos aquellos asociados con discapacidad motriz, en la Educacion Fisica.

En la pregunta número once, la información recabada es muy trascendental ya que posiblemente responda a la interrogante de preguntas anteriores, en donde los resultados revelan que en la ciudad de Cuenca el $45,8 \%$ de las instituciones educativas casi nunca cumplen con las normas básicas y que no cuentan con los implementos necesarios de trabajo utilizados en las clases de Educación Física dirigidos a estudiantes con discapacidad motriz; en tanto que apenas el 0,5\% exterioriza que cumplen con dichas normas y requerimientos; estos resultados estadísticos lo ratifica la UNESCO 
(2003) sustentando que, "la gran mayoría de los establecimientos de enseñanza son materialmente inaccesibles para muchos alumnos, especialmente los que tienen alguna discapacidad física". (p.11)

Para finalizar, la pregunta trece determina que, el 56,7\% de los docentes consideran muy necesario que las instituciones educativas cuenten con una guía de estrategias metodológicas para el área de Educación Física, con el fin de aplicar a estudiantes con discapacidad motora, ante lo expuesto Soto Calderón (2003) explica que las habilidades de enseñanza y aprendizaje deben estar dirigidas a todos los estudiantes de educación regular desarrollando el mismo plan curricular, teniendo en cuenta realizar los ajustes necesarios que requiera cada estudiante de acuerdo a sus necesidades, con el fin de lograr una participación activa en igualdad de condiciones, oportunidades y sobre todo fomentando el trabajo colaborativo entre los ellos.

\section{PROPUESTA}

Al finalizar el análisis de los resultados, ha sido posible determinar que el principal problema que tienen los docentes al trabajar con estudiantes asociados a la discapacidad motriz al momento de impartir las clases de Educación Física, es el desconocimiento de estrategias metodológicas y por ende la dificultad de planificación para las clases. En base a las dificultades detectadas, se propone las siguientes estrategias: 
Revista Arbitrada Interdisciplinaria KOINONIA

Año VI. Vol VI. N4. Edición Especial: Educación III. 2021

Hecho el depósito de Ley: FA2016000010 ISSN: 2542-3088

FUNDACIÓN KOINONIA (F.K). Santa Ana de Coro. Venezuela.

Edwin Geovanny Naranjo-Naranjo; Zoila Guillermina Torres-Palchisaca; Gema Barrachina-Fernández

Tabla 2.

Estrategias metodológicas aplicadas a estudiantes con discapacidad motriz.

\begin{tabular}{|c|c|c|}
\hline Estrategias & Procedimiento/aplicación & Objetivo \\
\hline $\begin{array}{l}\text { Sensibilización } \\
\text { sobre la } \\
\text { discapacidad } \\
\text { motriz. }\end{array}$ & $\begin{array}{l}\text { Socializar a los estudiantes regulares, } \\
\text { sobre la discapacidad que adolece } \\
\text { algún compañero de la clase. } \\
\text { Proyección de videos sobre la } \\
\text { clasificación de la discapacidad } \\
\text { motriz. }\end{array}$ & $\begin{array}{l}\text { Reconocer la importancia de la } \\
\text { diversidad e inclusión educativa, } \\
\text { fomentando la empatía y el } \\
\text { respeto entre la comunidad } \\
\text { educativa, para fortalecer la } \\
\text { equidad y la educación para todos. }\end{array}$ \\
\hline $\begin{array}{l}\text { Aceptar y valorar } \\
\text { las diferencias de } \\
\text { cada uno. }\end{array}$ & $\begin{array}{l}\text { Generar dinámicas grupales e } \\
\text { individuales. }\end{array}$ & $\begin{array}{l}\text { Reconocer a la discapacidad como } \\
\text { parte de la diversidad; } \\
\text { garantizando así que todos los } \\
\text { estudiantes tengan las mismas } \\
\text { oportunidades de participar en } \\
\text { todos los aspectos de la vida al } \\
\text { máximo de sus capacidades. }\end{array}$ \\
\hline $\begin{array}{l}\text { Interdependencia } \\
\text { positiva }\end{array}$ & $\begin{array}{l}\text { Actividades que motiven una } \\
\text { participación armónica individual. (El } \\
\text { estudiante guía el calentamiento, } \\
\text { asignación de roles, etc.) }\end{array}$ & $\begin{array}{l}\text { Concienciar a los estudiantes que } \\
\text { deben tener claro que los } \\
\text { esfuerzos de cada integrante no } \\
\text { sólo lo beneficia a él mismo sino } \\
\text { también a los demás. }\end{array}$ \\
\hline $\begin{array}{l}\text { Incorporar } \\
\text { objetos familiares } \\
\text { para los } \\
\text { estudiantes. }\end{array}$ & $\begin{array}{l}\text { Elaboración de implementos } \\
\text { deportivos con materiales reciclados y } \\
\text { la naturaleza. }\end{array}$ & $\begin{array}{l}\text { Generar continuamente } \\
\text { estimulación manipulativa y social, } \\
\text { aprovechando al máximo sus } \\
\text { recursos motrices. }\end{array}$ \\
\hline $\begin{array}{lr}\text { Fortalecer } & \text { las } \\
\text { actuaciones } & \text { e } \\
\text { incentivar } & \text { los } \\
\text { logros } & \text { de }\end{array}$ & $\begin{array}{l}\text { Frases de motivacion y exaltación: } \\
\text { "Muy bien", "Excelente", "Me gusta } \\
\text { cómo te esforzaste". }\end{array}$ & $\begin{array}{l}\text { Fomentar y crear relaciones de } \\
\text { afecto con los estudiantes, } \\
\text { propiciando de esta forma que el }\end{array}$ \\
\hline
\end{tabular}


Revista Arbitrada Interdisciplinaria KOINONIA

Año VI. Vol VI. N4. Edición Especial: Educación III. 2021

Hecho el depósito de Ley: FA2016000010

ISSN: 2542-3088

FUNDACIÓN KOINONIA (F.K). Santa Ana de Coro. Venezuela.

Edwin Geovanny Naranjo-Naranjo; Zoila Guillermina Torres-Palchisaca; Gema Barrachina-Fernández

\begin{tabular}{|c|c|c|c|}
\hline & $\begin{array}{l}\text { aprendizaje a los } \\
\text { estudiantes. }\end{array}$ & & $\begin{array}{l}\text { alumno realice actividades } \\
\text { armónicas y voluntarias. }\end{array}$ \\
\hline$>$ & $\begin{array}{l}\text { Realizar } \\
\text { actividades y } \\
\text { tomar como } \\
\text { referencia } \\
\text { temática que } \\
\text { sean de su } \\
\text { interés. }\end{array}$ & $\begin{array}{l}\text { Ejercicios y o/actividades que generen } \\
\text { más descanso a los estudiantes que } \\
\text { se les dificulte trabajar por un periodo } \\
\text { extenso. }\end{array}$ & $\begin{array}{l}\text { Motivar e incentivar a los } \\
\text { estudiantes la práctica y ejecución } \\
\text { de la actividad Fisica, para que } \\
\text { esta sea de su agrado y la } \\
\text { participación sea armónica. }\end{array}$ \\
\hline$>$ & $\begin{array}{l}\text { Organización } \\
\text { estratégica de los } \\
\text { estudiantes en la } \\
\text { clase. }\end{array}$ & $\begin{array}{l}\text { Ubicación del estudiante en lugares } \\
\text { acorde a las necesidades (adelante } \\
\text { del grupo, bajo techo), en los cuales } \\
\text { puedan asimilar de mejor manera la } \\
\text { clase. }\end{array}$ & $\begin{array}{l}\text { Garantizar las condiciones de } \\
\text { seguridad del estudiante al } \\
\text { momento de recibir la clase. }\end{array}$ \\
\hline$>$ & $\begin{array}{l}\text { Delimitación } \\
\text { adecuada del } \\
\text { espacio a utilizar. }\end{array}$ & $\begin{array}{l}\text { Adecuación del espacio (cintas, } \\
\text { círculos, césped, etc.) de acuerdo a } \\
\text { las necesidades de los estudiantes } \\
\text { con NEE y a las actividades } \\
\text { planificadas. }\end{array}$ & $\begin{array}{l}\text { Cumplir con las medidas de } \\
\text { seguridad pertinentes, para } \\
\text { garantizar su participación activa. }\end{array}$ \\
\hline$>$ & $\begin{array}{l}\text { Adaptación y } \\
\text { aplicación de } \\
\text { conocimientos a } \\
\text { situaciones } \\
\text { reales. }\end{array}$ & $\begin{array}{l}\text { Actividades y ejercicios adaptados a la } \\
\text { vida real. }\end{array}$ & $\begin{array}{l}\text { Fomentar en los estudiantes la } \\
\text { capacidad para identificar y } \\
\text { resolver un problema adaptado a } \\
\text { la vida real, empleando medidas } \\
\text { lógicas para encontrar } \\
\text { una solución deseada. }\end{array}$ \\
\hline & $\begin{array}{l}\text { Tutoría entre } \\
\text { compañeros }\end{array}$ & $\begin{array}{l}\text { Movimientos y ejercicios en los cuales } \\
\text { promuevan el desarrollo de } \\
\text { habilidades cognitivas-motrices, } \\
\text { producto de la interacción entre ellos. }\end{array}$ & $\begin{array}{l}\text { Incentivar al apoyo mutuo entre } \\
\text { compañeros, identificando las } \\
\text { dificultades, errores y aciertos } \\
\text { entre ellos para mejorar la } \\
\text { construcción del conocimiento. }\end{array}$ \\
\hline
\end{tabular}


Edwin Geovanny Naranjo-Naranjo; Zoila Guillermina Torres-Palchisaca; Gema Barrachina-Fernández

\begin{tabular}{|l|l|l|}
\hline $\begin{array}{l}\text { Prácticas lúdicas } \\
\text { e inclusivas }\end{array}$ & $\begin{array}{l}\text { Juegos grupales y cooperativos } \\
\text { Juegos de memoria y de mesa. }\end{array}$ & $\begin{array}{l}\text { Participar de manera placentera } \\
\text { cuidando de sí mismo y de los } \\
\text { demás. }\end{array}$ \\
\hline$>$ Musicoterapia & $\begin{array}{l}\text { Actividades con sonidos y música, } \\
\text { para promover la comunicación entre } \\
\text { compañeros y despertar emociones. }\end{array}$ & $\begin{array}{l}\text { Estimular y desarrollar } \\
\text { capacidades individuales, aparte } \\
\text { de fomentar la creatividad, } \\
\text { ejercitar la destreza manual y } \\
\text { autonomía del estudiante con } \\
\text { discapacidad motriz. }\end{array}$ \\
\hline $\begin{array}{l}\text { Aplicación de de } \\
\text { metodologías } \\
\text { aprendizas }\end{array}$ & $\begin{array}{l}\text { Aprendizaje cooperativo, ABP, } \\
\text { Enseñanza Reciproca, Gamificación y y } \\
\text { Aula invertida }\end{array}$ & $\begin{array}{l}\text { Desarrollar autonomía en los } \\
\text { estudiantes, con el propósito de } \\
\text { conseguir una mayor motivación } \\
\text { por el aprendizaje de nuevos } \\
\text { conocimientos y la adquisición de } \\
\text { nuevas habilidades en torno al } \\
\text { trabajo en equipo. }\end{array}$ \\
\hline $\begin{array}{l}\text { Evaluación } \\
\text { diferenciada }\end{array}$ & $\begin{array}{l}\text { Incorporar una evaluación del aporte } \\
\text { individual: física, escrita, verbal y/o } \\
\text { pictogramas. }\end{array}$ & $\begin{array}{l}\text { Identificar los niveles de logro de la } \\
\text { asignatura, garantizando el } \\
\text { proceso de enseñanza- } \\
\text { aprendizaje individual. }\end{array}$ \\
\hline
\end{tabular}

\section{CONCLUSIONES}

Una vez concluida la investigación y desarrollado el análisis respectivo de los resultados obtenidos del instrumento de investigación aplicado, se determina que:

Los problemas a nivel general de los docentes de Educación Física de la Ciudad de Cuenca, es sin lugar a duda la falta de aplicabilidad de estrategias metodológicas dirigidas a estudiantes con discapacidad motriz y la planificación de documentos curriculares adaptados (PUD, plan de clase), todo esto debido a la carencia de conocimientos, experiencia y experticia para trabajar idóneamente con estudiantes con NNE. 
No obstante, los docentes coincidieron que el Curriculo 2016 no se ajusta a las necesidades de aprendizaje y de igual forma no cuenta con los elementos necesarios para atender adecuadamente a estudiantes con necesidades educativas especiales, ante esta adversidad es importante que el docente reflexione y adquiera conocimientos constantes a través de la autocapacitación profesional, con la finalidad de brindar una educación de calidad y calidez a todos los estudiantes, propiciando de esta forma la inclusión educativa.

Para concluir, con esta investigación se generan posibles estrategias metodológicas (aplicación de metodologías activas de aprendizaje, evaluación diferenciada, musicoterapia) aplicadas a estudiantes con discapacidad motriz, en donde los docentes podrán guiarse con herramientas teóricas y prácticas frente a las demandas de los estudiantes con NNE en las instituciones educativas.

\section{REFERENCIAS CONSULTADAS}

Ana María Villón Tomalá1, K. A. (2001). La educación especial en la realidad ecuatoriana del siglo XXI. Fundacion Vidal, P.3.

Antonio Campo Peña, E. C.-M. (2020). Estrategias para la enseñanza de la educación física en búsqueda de la calidad educativa. Revista Cientifica de FAREM-Esteli , p.1.

Barros, S. F. (2021). Estrategias innovadoras para el proceso de enseñanza aprendizaje de la Educación Física en Bachillerato. Revista Arbitrada Interdisciplinaria Koinonía, 6(2), 25-50. doi: http://dx.doi.org/10.35381/r.k.v6i2.1223

Dabdub-Moreira, M., \& Pineda-Cordero, A. (2015). La atención de las necesidades educativas especiales y la labor docente en la escuela. Revista Costarricense de Psicología, p.42.

Dabdub-Moreira, M., \& Pineda-Cordero, A. (2015). La atención de las necesidades educativas especiales y la labor docente en la escuela primaria. Revista Costarricense de Psicología, p.43. 
Edgar Marcelo Méndez Urresta, J. B. (2018). El aprendizaje basado en problemas en la asignatura de didáctica de la educación física. Revista pedagógica de la Universidad de Cienfuegos, p.362.

Educación inclusiva y especial. (2011). Vicepresidencia de la república del ecuador, p.14.

El portal de contenidos en neurologia. (2021). Neurowikia, P.1.

Erazo Álvarez, J. C. (2021). Capital intelectual y gestión de innovación: Pequeñas y medianas empresas de cuero y calzado en Tungurahua-Ecuador. Revista De Ciencias Sociales, 27, 230-245. Recuperado a partir de https://n9.cl/7kn32

Erazo, J. y Narváez, C. Medición y gestión del capital intelectual en la industria del cuero - calzado en Ecuador. Revista Arbitrada Interdisciplinaria Koinonía, 5 (9), 437-467. Recuperado a partir de https://n9.cl/aeOhc

Grzona, M. A. (2014). La accesibilidad educativa en las aulas inclusivas. Una mirada didáctica. Investigación y Postgrado, p.140.

Guia de estrategias metodologicas para la Educacion Fisica en EGB y BGU. (2018). Ministerio de Educación del Ecuador, P.9.

Guía de trabajo adaptaciones curriculares para la educación especial e inclusiva. (2013). P.14.

Gutiérrez-Delgado, J. et al. (2018). Estrategias metodológicas de enseñanza y aprendizaje con un enfoque lúdico. Recuperado de https://n9.cl/8r623

Lobo Canella, J. M. (2018). La gamificación aplicada a la Educación Física en Primaria. Publicaciones didacticas, P.663.

Moreno Fergusson, P. A. (2009). Paraplejia pasado y futuro del ser. Index de Enfermería, p.2.

Cedeño-Escobar, J. A.-M. (2020). Aula invertida una estrategia motivadora de enseñanza para estudiantes de educación general básica. Ciencias de la Educación, P. 882. 
Carrión Macas, O. C. (2019). Inclusión educativa de las personas con necesidades educativas especiales permanentes. Universidad Técnica de Machala. Conrado, P.4.

Ministerio de Educacion . (2016). Curriculo de Currículo de EGB y BGU Eduacacion Fisica . p.8.

Navarro-Aburto, B. A., Arriagada Puschel, I. A., \& Osse-Bustingorry, S. (2016). Adaptaciones curriculares: Convergencias y divergencias de su implementación en el profesorado chileno. Revista Electrónica Educare , P.6.

Pappous, * . C. (2015). Actitudes de los profesionales de la educación . Revista Digital de Investigación Educativa, p.108.

Pascual, Á. F. (2011). La médula espinal: el cordón de la vida. Relato de un tetraplégico por accidente de tráfico. Index de Enfermería, p.2.

Petrou, O., \& Henríquez, A. (2006). Guía de juegos motrices, estrategia metodológica para desarrollar habilidades kinestésicas y coordinativas en niños de primer grado dirigida a docentes de Educación. Revista de Investigación, 145.

Soto Calderón, R. (2003). La inclusión educativa: Una tarea que le compete a toda una sociedad. Revista Electrónica "Actualidades Investigativas en Educación", p.12.

Taboada-Lugo, N., \& Minaya-Ramos, G. (2012). Caracterización clínica y etiológica de las diferentes discapacidades en el Estado Plurinacional de. Revista Peruana de Epidemiología, p.02.

Taboada-Lugo, N., \& Minaya-Ramos, G. (2012). Caracterización clínica y etiológica de las diferentes discapacidades en el Estado Plurinacional de Bolivia, 2009-2010. Revista Peruana de Epidemiología, p.01.

UNESCO. (1994). Declaración de Salamanca. Marco de acción para las necesidades educativas especiales. P.11.

UNESCO. (2003). Superar la exclusión mediante planteamientos integradores en la educación. Un desafío y una visión. P.11. 
Revista Arbitrada Interdisciplinaria KOINONIA

Año VI. Vol VI. N4. Edición Especial: Educación III. 2021

Hecho el depósito de Ley: FA2016000010 ISSN: 2542-3088

FUNDACIÓN KOINONIA (F.K). Santa Ana de Coro. Venezuela.

Edwin Geovanny Naranjo-Naranjo; Zoila Guillermina Torres-Palchisaca; Gema Barrachina-Fernández

Velázquez Callado, C., Fraile Aranda, A., \& López Pastor, V. M. (2014). Aprendizaje cooperativo en Educación Física. Movimento, p.240.

Zoila María Álava, R. F. (2019). Resiliencia en un grupo de discapacitados físico motores . Revista Cubana de Medicina General Integral, P.3-4.

C2021 por los autores. Este artículo es de acceso abierto y distribuido según los términos y condiciones de la licencia Creative Commons Atribución-NoComercial-Compartirlgual 4.0 Internacional (CC BY-NC-SA 4.0)

(https://creativecommons.org/licenses/by-nc-sa/4.0/).x| 